\title{
Grounding-Mechanical Explanation
}

\author{
Kelly Trogdon, Virginia Tech \\ Forthcoming, Philosophical Studies
}

\begin{abstract}
I argue that there is an important similarity between causation and grounding. In particular I argue that, just as there is a type of scientific explanation that appeals to causal mechanisms - causal-mechanical explanation - there is a type of metaphysical explanation that appeals to grounding mechanisms-grounding-mechanical explanation. The upshot is that the role that grounding mechanisms play in certain metaphysical explanations mirrors the role that causal mechanisms play in certain scientific explanations. In this light, it becomes clear that grounding-mechanical explanations make crucial contributions to the evaluation of a variety of important philosophical theses, including priority monism and physicalism.
\end{abstract}

\section{Introduction}

There is a growing literature on grounding, which some claim is a distinctive form of determination important to metaphysics and philosophy in general. For the purposes of this article I assume that grounding talk is best regimented with a predicate understood as expressing a relation holding between facts. As for facts, I assume what Fine (1982) calls objectualism — a fact is a complex entity with the entities it concerns as constituents. So the fact that Socrates is a philosopher, for example, has Socrates as well as being a philosopher as constituents. ${ }^{1}$ By 'grounding' I always mean full grounding unless otherwise noted, where intuitively a full ground is enough on its own to ground what it grounds, and a mere partial ground isn't enough on its own to ground what it grounds. ${ }^{2}$

Discussions of grounding typically focus on either clarifying the concept (e.g. arguing that grounding isn't transitive) or applying the concept (e.g. arguing that certain philosophical theses such as physicalism are profitably formulated in terms of

\footnotetext{
${ }^{1}$ Just how to understand composition in this context is a topic that I won't explore here. Armstrong (1997), for example, argues that the relevant notion isn't unique in the sense that two facts can have the same constituents. I also work with what Fine calls a worldly conception of facts - roughly speaking, facts don't have representations or modes of presentation as constituents - and I assume that facts are a distinctive kind of entity.

${ }^{2}$ For a general discussion of grounding that touches on these assumptions and related issues, see Trogdon (2013a).
} 
grounding). ${ }^{3}$ My project here is chiefly one of clarification rather than application. One way to clarify the nature of something is to show how it's related to other notions that we have a relatively firm grip on. And one notion of philosophical interest that we have a facility with is that of causation. So the following question arises: how exactly are grounding and causation related to one another?

Schaffer (2016a) and A. Wilson (forthcoming) argue that, just as causal relationships are usefully represented by systems of equations and directed graphs, so too are grounding relationships. Indeed, Wilson argues that grounding is a species of causation partly on this basis. I focus, however, on a different potential connection between causation and grounding. I begin by characterizing what I call grounding mechanisms. In short, grounding mechanisms are determination relations of a certain sort holding between constituents of grounding facts and constituents of the facts they ground. Then I argue that, just as there is a type of scientific explanation that appeals to causal mechanisms - causal-mechanical explanation-there is a type of metaphysical explanation that appeals to grounding mechanisms-groundingmechanical explanation. In short, grounding-mechanical explanations are representations of grounding relations as being instances of grounding mechanisms. The upshot is that the role that grounding mechanisms play in certain metaphysical explanations mirrors the role that causal mechanisms play in certain scientific explanations. In this light, it becomes clear that grounding-mechanical explanations make crucial contributions to the evaluation of a variety of important philosophical theses, including priority monism and physicalism. ${ }^{4}$

\section{Mechanisms}

\subsection{Metaphysical determination relations}

I understand the notion of a grounding mechanism in terms of what I call metaphysical determination relations. What are these relations, and what unifies them so that they're deserving of a common name? I'll consider the issue of unification first. I propose that these relations are unified in that they're essentially connected to grounding in the same way.

\footnotetext{
${ }^{3}$ See Schaffer (2012) for an argument that grounding (understood as binary relation) isn't transitive, and Dasgupta (2015) for a discussion of grounding-theoretic formulations of physicalism.

${ }^{4}$ While I won't consider the details of their proposals, I take it that what I go on to say about grounding is broadly compatible with both Schaffer's and Wilson's views mentioned above. While I'm arguing that grounding is substantively like causation in one key respect, see Bernstein (forthcoming) and Koslicki (2016) for arguments that there are important respects in which they're dissimilar. They don't address, however, mechanisms in particular.
} 
Suppose that a relation $\mathrm{R}$ satisfies the following condition: if the $\mathrm{xs}$ stand in $\mathrm{R}$ to $\mathrm{y}$ then there is some fact $\mathrm{A}$, some fact $\mathrm{B}$, and some plurality of facts $\Delta$ such that the xs are constituents of $\mathrm{A}, \mathrm{y}$ is a constituent of $\mathrm{B}, \mathrm{A}$ is among $\Delta$, and $\Delta$ grounds $\mathrm{B}$. Call this the grounding condition. I stipulate that $\mathrm{R}$ is a metaphysical determination relation just in case the foregoing is an essential truth about $\mathrm{R}$. In other words, $\mathrm{R}$ is a metaphysical determination relation just in case satisfying the grounding condition is part of what it is to be R. ${ }^{5}$ Let's consider some examples of what I take to be metaphysical determination relations, which will make the proposal easier to understand.

I think that set formation and the determinate-determinable relation are metaphysical determination relations. So I propose that for each of these relations it's an essential truth about that relation that it satisfies the grounding condition. In particular:

- Set formation: part of what it is to be set formation is that if $\mathrm{x}$ (e.g. Socrates) stands in this relation to $y$ (e.g. \{Socrates\}) then the fact that $\mathrm{x}$ exists grounds the fact that y exists (e.g. the fact that Socrates exists grounds the fact that \{Socrates\} exists).

- The determinate-determinable relation: part of what it is to be the determinate-determinable relation is that if $\mathrm{P}$ (e.g. being burgundy) stands in this relation to $\mathrm{Q}$ (e.g. being red) on an occasion such that $\mathrm{x}$ has $\mathrm{P}$ and $\mathrm{Q}$, then the fact that $\mathrm{x}$ has $\mathrm{P}$ grounds the fact that $\mathrm{x}$ has $\mathrm{Q}$ (e.g. the fact that $\mathrm{x}$ is burgundy grounds the fact that $\mathrm{x}$ is red).

In the case of set formation and the determinate-determinable relation, the corresponding grounding facts are full grounds - the fact that Socrates exists is enough on its own to ground the fact that \{Socrates\} exists, and the fact that $\mathrm{x}$ is burgundy is enough on its own to ground the fact that $\mathrm{x}$ is red.

There are other metaphysical determination relations, however, such that the corresponding grounding facts aren't enough on their own to ground what they ground-they're mere partial grounds. Consider, for example, constitution, functional realization, and mereological realization. These relations in my view are metaphysical determination relations. So I think that for each of these relations it's

\footnotetext{
${ }^{5}$ Following Fine (1994), it's an essential truth about some entity that p just in case part of what it is to be that entity is that p. Perhaps it's an essential truth about Socrates that if Socrates exists then Socrates is a human. While it's a necessary truth that if Socrates exists then $1+1=2$, this truth isn't an essential truth about Socrates- the nature of Socrates "knows nothing" of arithmetic.
} 
an essential truth about that relation that it satisfies the grounding condition. In particular:

- Constitution: part of what it is to be constitution is that if $\mathrm{x}$ (e.g. the lump of clay) stands in this relation to $y$ (e.g. the statue) then the fact that $x$ exists is among some plurality of facts that grounds the fact that y exists (e.g. the fact that the lump of clay exists is among some plurality of facts that grounds the fact that the statue exists).

- Functional realization: part of what it is to be functional realization is that if $\mathrm{P}$ (e.g. being a DNA molecule) stands in this relation to Q (e.g. being a gene) on an occasion such that $\mathrm{x}$ has $\mathrm{P}$ and $\mathrm{Q}$, then the fact that $\mathrm{x}$ has $\mathrm{P}$ is among some plurality of facts that grounds the fact that $\mathrm{x}$ has $\mathrm{Q}$ (e.g. the fact that $\mathrm{x}$ is a DNA molecule is among some plurality of facts that grounds the fact that $\mathrm{x}$ is a gene).

- Mereological realization: part of what it is to be mereological realization is that if the Ps (e.g. certain molecular properties) stand in this relation to Q (e.g. the property of being hard) on an occasion such that the xs have the Ps, $y$ has $\mathrm{Q}$, and the xs compose $\mathrm{y}$, then the fact that the xs compose $\mathrm{y}$ and have the Ps is among some plurality of facts that grounds the fact that $y$ has Q (e.g. the fact that the xs compose y and have thus-and-so molecular properties is among some plurality of facts that grounds the fact that $y$ is hard).

It's not essential for our purposes that we settle here just what facts we might add to the mere partial grounds in these cases to get full grounds. In the case of constitution, if $\mathrm{x}$ is a lump of clay and $\mathrm{y}$ is a statue, then perhaps the supplementing facts concern how the clay and statue are regarded. In the case of functional realization, if $\mathrm{P}$ is the property of being a DNA molecule and Q the property of being a gene, then the supplementing facts concern at the very least causal details of the chemical system in which the molecule is embedded. And in the case of mereological realization, if the Ps are various molecular properties and Q is the property of being hard, then again the supplementing facts concern at the very least causal details of the chemical system in which the relevant molecules are embedded. ${ }^{6}$

\footnotetext{
${ }^{6}$ It may be that some relations satisfy the grounding condition yet it isn't part of their nature to do so. Suppose that constitution and relation $\mathrm{R}$ are distinct but necessarily co-extensive. As the former satisfies the grounding condition, so too does the latter. Yet it's not part of what it is to be $\mathrm{R}$, we will suppose, that it satisfies this condition- the nature of this relation "knows nothing" of grounding. So R isn't a metaphysical determination relation despite the fact that it satisfies the grounding condition.
} 
Before moving on to grounding mechanisms, a final thought. You might argue that grounding is a justified theoretical posit as follows: thus-and-so metaphysical relations are importantly unified in that they share various features, and this gives us reason to think that there is a distinctive metaphysical relation-the grounding relation-that unifies them. Now, in addition to being essentially connected to grounding in the same way, the relations I discuss above share other features as well, ones that don't concern grounding per se. Each relation, for example, takes constituents of facts rather than facts themselves as relata, holds non-diachronically, and is generative, irreflexive, and asymmetric. While I'm going to suppose that all metaphysical determination relations have these features, I don't wish to argue that we're justified in positing grounding on the basis of these shared features. ${ }^{7}$ Instead, I claim that, given the working assumption that there is a grounding relation, these relations are plausibly viewed as having a particular feature concerning grounding in common-they're essentially connected to grounding in the same way. ${ }^{8}$

\subsection{Grounding mechanisms}

I can now characterize the notion of a grounding mechanism as well as what it is for a grounding relation to be an instance of a grounding mechanism. A grounding mechanism is either a metaphysical determination relation or a chain of such relations. Supposing that $\Delta$ grounds $\mathrm{A}$, this grounding relation is an instance of a grounding mechanism just in case there is some fact among $\Delta$ with a constituent(s) that stands in a metaphysical determination relation or a chain of such relations to some constituent of A. But why call metaphysical determination relations or chains

\footnotetext{
${ }^{7}$ Koslicki (2015) and Wilson (2014) consider unification-style arguments for grounding and reject them. They (independently) argue that the relevant metaphysical relations don't seem to display the sort of unity required for us to be justified in positing a distinctive metaphysical relation as a unifier. And they (again independently) argue that, even if the relevant metaphysical relations turn out to be unified in an important way, this on its own might not license the claim that there is a distinctive metaphysical relation that unifies them in any case.

8 There is a substantial literature on each of the relations I've discussed in this section. For overviews of the literature on the determinate-determinable relation and constitution, see J. Wilson (2017) and Wasserman (2015), respectively. For an overview of the literature on functional and mereological realization, see Baysan (2015). (For realization aficionados, I'm open to the idea that there are still other forms of realization, such as subset realization as characterized by Wilson (1999) and others, and that these likewise count as metaphysical determination relations in my sense.) For influential discussions of set formation, see Fine (2010a) and Lewis (1991, Ch. 1). Some characterizations of these relations proposed in the literature are compatible with my claim that they're essentially connected to grounding-see, e.g., Doepke (1999, Ch. 7) on constitution. Indeed, some of these characterizations seem to directly appeal to grounding - see, e.g., Gillett (2007) on mereological realization. Other such characterizations, however, on the face of it are incompatible with the essence claim-see, e.g., Lewis on set formation.
} 
of such relations 'mechanisms'? The answer is that representations of causal relations as being instances of causal mechanisms are potentially informative in that they communicate facts about "how things work" (Craver, 2007, 110), and representations of grounding relations as being such that constituents of their relata stand in metaphysical determination relations or chains of such relations are potentially informative in the same sense. We will return to this comparison in the next section.

Importantly, I'm not aiming to analyze the notion of grounding in terms of grounding mechanisms - the analysis goes in the other direction. It's potentially instructive to compare what's going on here with how similar issues are treated in the literature on causal mechanisms. To the extent that there is an orthodox view about causal mechanisms it's the system view according to which a causal mechanism is a complex arrangement of parts that is responsible for some phenomenon, partly in virtue of the organization of those parts. As Machamer et al describe the view, causal mechanisms are composed of "...entities and activities organized such that they are productive of regular changes from start or set-up to finish or termination conditions" $(2000,3)$. Here the "start" is a cause of the "finish", and "activities" are types of causes. So advocates of the system view aim to analyze causal mechanisms in terms of causation rather than the other way around. ${ }^{9}$

Consider the following grounding claims: the fact that Socrates exists grounds the fact that $\{$ Socrates $\}$ exists; the fact that $\mathrm{x}$ is burgundy grounds the fact that $\mathrm{x}$ is red; the fact that the clay exists (together with other facts) grounds the fact that the statue exists; the fact that $x$ is a DNA molecule (together with other facts) grounds the fact that $x$ is a gene; and the fact that the xs compose $y$ and have thus-and-so molecular properties (together with other facts) grounds the fact that $y$ is hard. In each of these cases a single metaphysical determination relation (set formation, the determinatedeterminable relation, constitution, functional realization, and mereological realization, respectively) connects constituents of the relevant facts rather than chains of such relations. This comports with the idea that these are cases of immediate rather than mediate grounding. In cases of immediate grounding there is no room, so

\footnotetext{
${ }^{9}$ The main rival to the system view of causal mechanisms is the process view. Railton claims that, while a scientific explanation of some event may include reference to a covering law, it must be supplemented by "an account of the mechanism(s) at work," where an account of a mechanism is "a more or less complete filling-in of the links in the causal chains" (1978, 748). Proponents of the process view, following Railton, conceive of causal mechanisms as concrete physical processes that transfer marks, mass-energy, or some other conserved physical quantity. Some such as Salmon (1984) and Dowe (1992) go on to propose reductive accounts of causation in terms of causal mechanisms so understood.
} 
to speak, for the chaining of metaphysical determination relations between constituents of the relevant facts - there is only room for one such relation.

There are also cases of grounding that involve the chaining of metaphysical determination relations. Suppose that the fact that thus-and-so nucleotides compose $\mathrm{x}$ and have thus-and-so properties (together with other facts) grounds the fact that $\mathrm{x}$ is a gene. In this case the nucleotide fact (together with other facts) grounds the fact that $\mathrm{x}$ is a DNA molecule which in turn (together with other facts) grounds the gene fact. And in this case there is a chain of metaphysical determination relations connecting constituents of the relevant facts. The properties of the nucleotides (constituents of the first fact) mereologically realize the property of being a DNA molecule (a constituent of the second fact) on this occasion, and the property of being a DNA molecule functionally realizes the property of being a gene (a constituent of the third fact) on this occasion.

The chain of metaphysical determination relations in this case involves tokens of type distinct relations. So, even if mereological and functional realization are transitive, a transitive inference to the conclusion that the properties of the nucleotides stand in a metaphysical determination relation to the property of being a gene on this occasion isn't licensed. Still, it may be that these properties stand in a metaphysical determination relation to the property of being a gene on this occasion. If they are so related then this case arguably involves both mediate and immediate grounding. ${ }^{10}$

A final point of clarification about grounding mechanisms concerns bare grounding, grounding relations that aren't instances of grounding mechanisms. Advocates of the system view of causal mechanisms typically maintain that, while the causal relations we encounter in the non-fundamental physical and social sciences are instances of causal mechanisms, it may be that the causal relations involved in fundamental physics aren't instances of causal mechanisms. For example, given the system view what causal mechanisms might be operative in instances of gravitational attraction? Likewise, I wish to leave open for now the possibility of bare grounding - it may be that some grounding relations aren't instances of grounding mechanisms.

If causation in fundamental physics challenges the idea that all causal relations are instances of causal mechanisms, where can we find grounding that challenges the idea that all grounding relations are instances of grounding mechanisms? A good place to look is the domain of conceptual and logical truths. Suppose the fact that $x$

${ }^{10}$ See Fine (2012) for discussion of similar cases_ cases that seem to involve both mediate and immediate grounding - and the complications they pose for characterizing the immediate/mediate grounding distinction. 
is red and the fact that $\mathrm{y}$ is round ground the conjunctive fact that $\mathrm{x}$ is red and $\mathrm{y}$ is round. (Here we're in the domain of logical truths - it's a logical truth that if A obtains and B obtains then A\&B obtains.) Many proponents of grounding accept such grounding claims, and they're sometimes referred to as cases of conceptual grounding. Now, it's clear that conjunction introduction itself isn't a metaphysical determination relation-if it's a relation at all (as opposed to an operation) then it takes propositions rather than objects or properties as its relata. On the face of it no metaphysical determination relation or chain of such relations connects constituents of either the red fact or the round fact to constituents of the conjunctive fact. Similar considerations apply to grounding claims that correspond to other inference rules such as existential and disjunction introduction. We will return to bare grounding later.

\section{Explanation}

\subsection{Grounding-mechanical explanation}

Above I explained what I mean by grounding mechanisms and what it is for a grounding relation to be an instance of a grounding mechanism. Now it's time to turn to explanation. I'm going to work with what we can call the bybrid view according to which explanations have both ontic and epistemic dimensions. On this view explanations consist of propositions or other abstract structures that together accurately and informatively represent portions of the world's structure. This contrasts with the epistemic view according to which explanations are merely informative representations, and the ontic view according to which explanations just are portions of the structure of the world. ${ }^{11}$

Turning to scientific explanation in particular, the causal mechanism view says that some scientific explanations are representations of causal relations as being instances of causal mechanisms. With this in mind, we can formulate a corresponding view with respect to metaphysical explanation. According to the grounding mechanism view, some metaphysical explanations are representations of grounding relations as being instances of grounding mechanisms. Just as we get different versions of the causal mechanism view depending on our views about causal mechanisms, we get different versions of the grounding mechanism view depending on our views about grounding mechanisms. Let's henceforth understand the causal mechanism view as appealing to the system view of causal mechanisms in particular, and the grounding mechanism

${ }^{11}$ See Salmon (1989) for an influential discussion of views similar to what I call the ontic and epistemic views, and Kim (1994) and Ruben (2012, Ch. 5) for more on what I'm calling the hybrid view. 
view as appealing to the conception of grounding mechanisms developed in the previous section. ${ }^{12}$

General considerations about explanation support the grounding mechanism view. Our theorizing about causation should concern not only what causes what but also how particular causal transactions take place-it should provide us with some guidance in answering questions like "How did this event cause that one?" Schaffer (2016b) reasonably claims that something similar is true with respect to grounding. Our theorizing here should concern, among other things, "how the connection runs" when some facts ground another-it should provide us with some guidance in answering questions like "How do these facts ground that one?" $(151)^{13}$

Why is it, however, that we're interested in how instances of causation and grounding unfold in the first place? With respect to causation, the answer, I take it, is quite simple —we're interested in providing explanations, and representations of how particular causal transactions take place are potentially informative in a distinctive way. As Bechtel and Abrahamsen put the idea, scientists typically "explain why by explaining how" (2005, 422). As Machamer et al put the idea, "To explain is not merely to redescribe one regularity as a series of several." It is, for example, “...the unwinding, bonding, and breaking that explain the protein synthesis; it is the binding, bending, and opening that explain the activity of NA+ channels" (2000, 20-21).

I want to suggest that corresponding considerations apply to grounding-exhibiting "how the connection runs" between grounding facts and the facts they ground is potentially informative in a distinctive way. It's the straightforward connection between how-questions regarding causation on the one hand and explanation and why-questions on the other that motivates the constraint on theorizing about causation mentioned above, and I propose that corresponding considerations motivate Schaffer's constraint on theorizing about grounding.

So far, so good. But what is it to exhibit how some causal or grounding transaction takes place on a particular occasion? Well, scientists - particularly those in biological and chemical sciences - explain how causation takes place by providing causalmechanistic models. According to Bechtel and Abrahamsen, such a model describes

\footnotetext{
12 While I assume that there are both scientific and metaphysical explanations, I won't try to characterize the scientific part of the former or the metaphysical part of the latter-see Kovacs (forthcoming) and Schaffer (2016a) for discussion.

${ }^{13}$ I don't mean to suggest that for every causal relation there is a substantive answer to the question of how the causation takes place in that case, and the same goes for grounding. The claim instead is that our theorizing about causation/grounding should help us provide substantive answers to these sorts of questions when there are such answers to be had.
} 
"...relevant component parts and operations, the organization of the parts and operations into a system, and the means by which operations are orchestrated so as to produce the phenomenon" $(2005,425)$. Kaplan and Bechtel state, "What is required to explain [in the relevant sense] a given phenomenon is to identify the responsible mechanism and the conditions under which it is operating." The task is to look "beneath the regularities couched at the behavioral level to reveal underlying mechanisms" (2011, 442).

As Franklin-Hall (2016) notes, not just any (accurate) representation of a causal relation as being an instance of a causal mechanism is explanatorily apt. For example, a model that represents a system as being an opaque black box won't do. So a mechanistic model of a causal relation must include information about the internal structure of the relevant system. ${ }^{14}$ Suppose, for example, that the exposure of the dendrites of a neuron to neurotransmitters causes neurotransmitters to be released at its axon terminal. A mechanistic model of this causal relation will include information about how the neuron has various organized macro-molecular parts that causally interact with one another in systematic ways. These parts include membranes, channels within them, and ionic concentrations in the internal and external environment. With recourse to this model we can see what went on "under the hood" in this case. ${ }^{15}$

Turning to grounding, suppose that the fact that the cut diamond is hard is partially grounded in the fact that its constituent carbon atoms are bonded and spatially arranged in thus-and-so way. How does the connection run in this case? We can answer this question by providing a mechanistic model of this grounding relation, a model according to which it's an instance of a grounding mechanism. ${ }^{16}$ The model in this case involves mereological realization. Let's follow Gillett (2007) and understand mereological realization in terms of the idea that some causal powers are comprised

\footnotetext{
${ }^{14}$ See Bechtel and Abrahamsen (2005), Craver (2007, Ch. 5), and Glennan (2005) for further discussion of constraints for mechanistic models of causal relations.

${ }^{15}$ Here the focus is causal mechanisms and horizontal causal relations, roughly causal relations connecting events involving entities occupying the same level of mereological aggregation. I should note, however, that there is growing interest in how causal mechanisms are related to vertical causation and other relations that involve movement across levels of mereological aggregation. Just how to think about the relationship between causal mechanisms and vertical causation and other vertical relations (and what implications this might have for grounding) is an interesting matter, but I won't pursue it further here-see Aizawa and Gillett (2016b) for discussion.

${ }^{16}$ Schaffer (2016b) sees his interventionist approach as telling us "how the connection runs" in cases of grounding, as it provides structural equations that encode counterfactual truths with respect to grounds and what they ground (e.g. had Socrates not existed, \{Socrates\} wouldn't have either).
} 
of other causal powers. Roughly speaking, we can say that if the xs have the Ps and y has Q then the Ps mereologically realize Q on this occasion just in case the xs compose y and the causal powers that the Ps contribute to the xs comprise the causal powers that $\mathrm{Q}$ contributes to $\mathrm{y}$, where $\mathrm{Q}$ itself is individuated by its causal powers.

Returning to the diamond, our mechanistic model of the grounding relation in this case will say at least three things. First, there are carbon atoms that compose the diamond, and have particular properties that are constituents of the grounding fact and bestow certain causal powers to the atoms. Second, the property of being hard is a constituent of the grounded fact and is individuated by its causal powers that it bestows to the diamond. Third, the causal powers bestowed to the carbon atoms comprise the causal powers bestowed to the diamond. With recourse to this model we can see just how the fact that the carbon atoms compose the diamond and are bonded and spatially arranged in thus-and-so way grounds the fact that the diamond is hard.

To return to a case that we've already considered, suppose again that the fact that thus-and-so nucleotides compose $\mathrm{x}$ and have thus-and-so properties partially grounds the fact that $\mathrm{x}$ is a gene. How does the connection run in this case? Again, we can answer this question by providing a mechanistic model for this grounding relation. The model in this case appeals to both functional and mereological realization. As is standard, let's understand functional realization in terms of causal roles. Roughly speaking, we can say that if $\mathrm{x}$ has $\mathrm{P}$ and $\mathrm{Q}$ then $\mathrm{P}$ functionally realizes $\mathrm{Q}$ on this occasion just in case $\mathrm{Q}$ is the property of having some property or other, instances of which play thus-and-so causal role, and the instance of $\mathrm{P}$ that characterizes x plays that causal role.

Our mechanistic model of the grounding relation in this case will say at least five things. First, there are nucleotides that compose $\mathrm{x}$ and have particular properties that are constituents of the grounding fact and bestow certain causal powers to those molecules. Second, the property of being a DNA molecule is a constituent of the fact that $\mathrm{x}$ is a DNA molecule, and it is individuated by its causal powers that it bestows to $\mathrm{x}$. Third, the causal powers bestowed to the nucleotides comprise the causal powers bestowed to $\mathrm{x}$. Fourth, the instance of the property of being a DNA molecule that characterizes x plays causal role R. Fifth, the property of being a gene is the property of having some property or other, instances of which play $\mathrm{R}$, and this property is a constituent of the grounded fact. With recourse to this model we can see just how the fact that thus-and-so nucleotides compose $\mathrm{x}$ and have thus-and-so properties partially grounds the fact that $\mathrm{x}$ is a gene. 
So the idea in a nutshell is this: specifying how the connection runs between grounding facts and facts they ground is potentially informative in a distinctive way, and one way of providing such specifications is to provide mechanistic models of grounding relations, models according to which they're instances of grounding mechanisms. This is why some metaphysical explanations are representations of grounding relations as being instances of grounding mechanisms-we can call such representations grounding-mechanical explanations.

I've argued that when it comes to grounding it's important to think about how grounding relations are instantiated. But note that some metaphysical determination relations are such that we can coherently ask how those relations are instantiated as well. Polger (2010), for example, argues that realization is like this. Suppose we have a mechanistic model of a grounding relation where realization (either functional or mereological) is the relevant metaphysical determination relation. Must this model specify just how properties are realized on this occasion in order to be explanatory? I don't think so-it seems that it's enough to show how some facts ground another fact to show that property constituents of the grounding facts realize property constituents of the grounded fact — we needn't also address how the former realize the latter. Compare: explanatory models of causal mechanisms typically abstract away from various details, and one such detail concerns just how certain causal relations between the working parts of the target mechanism unfold, many of which themselves are instances of further causal mechanisms (Craver 2006). For example, while causal mechanisms for strong and weak chemical bonds are components of the mechanisms of replication, transcription, and translation of DNA and RNA, respectively, and the latter in turn are components of mechanisms for various cell activities, molecular biologists typically don't engage with the details of just how chemical bonding takes place. This is the point at which mechanical description "bottoms out" for molecular biologists given their interests (Machamer et al 2000). The moral is that it's enough to show how a causal relation unfolds to show that the working parts of the relevant mechanism causally interact in particular ways-we needn't also address how each of the causal interactions between the parts take place.

\subsection{Grounding mechanisms vs. metaphysical subsumption}

The goal of this section is to further clarify the grounding mechanism view by showing how it's an alternative to a view according to which all metaphysical explanations appeal to metaphysical laws. It will help to begin by considering corresponding issues with respect to scientific explanation. According to the nomic subsumption view, any scientific explanation is a representation according to which certain events in conjunction with certain laws logically entail certain events. On this view, some scientific explanations concern causation in particular-they're 
representations according to which certain events cause, and in conjunction with certain laws logically entail, certain events. The causal mechanism view is an alternative to the nomic subsumption view. A crucial point of contrast between these views is that, while the nomic subsumer claims that all scientific explanations involve the representation of laws, the causal mechanist denies that this is so. ${ }^{17}$

Granting for the sake of argument that there are laws in biology—see Mitchell (2000) for a case that there are "pragmatic" biological laws_it seems fairly clear that some of the explanations that biologists provide don't appeal to them. ${ }^{18}$ As Craver and Kaiser (2013) point out, explanatory models of causal mechanisms include exemplars, where the intended scope of such a model isn't part of that model's content. For example, the content of a labeled diagram of a causal mechanism for neurotransmitter release working from beginning to end concerns representative instances rather than general types. So the idea is that some scientific explanations are models of causal mechanisms, and some of these models don't themselves appeal to laws or even generalizations. Note that consistent with the causal mechanism view, however, is the idea that some scientific explanations appeal to covering laws rather than causal mechanisms, and that some mechanistic models of causal relations incorporate representations of laws that characterize the causal interactions among the working parts of the target mechanisms.

According to the metaphysical subsumption view, any metaphysical explanation is a representation according to which certain facts in conjunction with certain metaphysical laws logically entail certain facts. On this view, some metaphysical explanations concern grounding in particular - they're representations according to which certain facts ground, and in conjunction with certain metaphysical laws logically entail, certain facts. Just as the causal mechanism view is an alternative to the nomic subsumption view, the grounding mechanism view is an alternative to the metaphysical subsumption view. And, similar to what I said above, an important point of contrast between these views is that, while the metaphysical subsumer

\footnotetext{
${ }^{17}$ In addition, some causal mechanists claim that (i) certain phenomena that the nomicsubsumer claims are explained by laws are in instead explained by causal mechanisms (Anderson 2011), (ii) some of what we take to be law-statements are instead descriptions of causal mechanisms (Glennan 2002), and (iii) causal mechanisms explain or sustain certain laws or law-like entities (Bechtel and Abrahamsen 2005; Cummins 2000; Glennan 2005).

18 Provided that there are no biological laws as some such as Beatty (1995) argue, since there are biological explanations it follows that not all scientific explanations involve the representation of laws.
} 
claims that all metaphysical explanations involve the representation of laws, the grounding mechanist denies that this is so. ${ }^{19}$

Granting for the sake of argument that there are metaphysical laws, I think it's fairly clear that there are metaphysical explanations that don't appeal to them. The two examples of grounding-mechanical explanations discussed above involving the diamond and nucleotides are examples — metaphysical laws aren't included in the contents of the relevant representations in these cases. So the idea is that some metaphysical explanations are models of grounding mechanisms, and some of these models don't themselves appeal to metaphysical laws. Note that consistent with the grounding mechanism view, however, is the idea that some metaphysical explanations appeal to covering laws rather than grounding mechanisms, and that some mechanistic models of grounding relations incorporate representations of metaphysical laws that characterize the metaphysical determination relations holding between constituents of grounding facts and constituents of the facts they ground.

The causal mechanist claims that it's enough for a representation to be explanatory that it correctly describes the causal structures that produce, underlie, or maintain the explanandum. Similarly, the grounding mechanist claims that it's enough for a representation to be explanatory that it correctly describes the underling metaphysical determination relations that link constituents of grounding facts with constituents of the facts they ground. ${ }^{20}$

${ }^{19}$ Here's another potential contrast between the grounding mechanism and metaphysical subsumption views. As we have seen, there is a systematic connection between the patterns of instantiation of relations like set formation between entities on the one hand and grounding relations between facts with those entities as constituents on the other. What explains this connection? The metaphysical subsumer might claim that the metaphysical laws explain why these relations and grounding are so connected (Wilsch 2015). Note that the grounding mechanist, by contrast, can appeal to the notion of a grounding mechanism to explain this connection. According to the grounding mechanist, part of what it is to be a relation like set formulation is that it's systematically connected to grounding (i.e. part of what it is to be set formation is that if $\mathrm{x}$ stands in this relation to $\mathrm{y}$ then the fact that $\mathrm{x}$ exists grounds the fact that y exists).

${ }^{20}$ Kment (2014, Ch. 6) and Wilsch $(2015,2016)$ endorse covering law conceptions of metaphysical explanation. There is a wrinkle here, however - they endorse the ontic view of explanation rather than the hybrid view, pushing explanations out into the world. Wilsch argues that for some facts to metaphysically explain another fact just is for the former together with the metaphysical laws to logically entail the latter in the right way. And Kment claims that any metaphysical explanation is such that its explanans logically entails its explanandum, where the explanans consists of a ground of the explanandum and a metaphysical law linking the ground to the explanandum. Just what the grounding mechanism view would look like cast in ontic terms and how it compares to the views of Kment and Wilsch is an interesting matter, but I won't pursue it further here. My general 


\subsection{A potential problem}

Earlier we saw that conceptual grounding challenges the idea that all grounding relations are instances of grounding mechanisms. What I've said about groundingmechanical explanation is compatible with bare grounding, so conceptual grounding insofar as it points to bare grounding isn't problematic. There is, however, a potential problem for the grounding mechanism view concerning cases of conceptual grounding in which the grounding relations are instances of grounding mechanisms.

Suppose that, while \{Socrates\} doesn't have property P, the fact that Socrates exists grounds the fact that Socrates exists or \{Socrates\} has P. A constituent of the grounding fact (Socrates) stands in a metaphysical determination relation (set formation) to a constituent of the grounded fact (\{Socrates $\}$ ). It follows that the grounding relation in this case is an instance of a grounding mechanism. What's the problem? Well, above I claim that one way of specifying how the connection runs with a grounding relation is to provide a model of the relation according to which it's an instance of a grounding mechanism. But it seems that by representing the grounding relation in the Socrates case as being an instance of a grounding mechanism involving set formation we don't show how the grounding connection runs. If it's proper to speak of a way or manner in which the grounding unfolds here, it presumably concerns disjunction introduction rather than set formation.

How to respond to this problem? Provided that the problematic cases are restricted to cases of conceptual grounding, one option is to simply deny that there are cases of conceptual grounding to begin with. While this goes against current orthodoxy, I can think of two potential reasons for going in this direction. First, there are various puzzles and paradoxes concerning grounding that have been developed in the literature — see Clark (2015), Fine (2010b), and Litland (2015)—and, while I won't go into the details, each one essentially appeals to conceptual grounding. So if there is no such thing as conceptual grounding then these various problems don't arise. All other things being equal, a regimentation of grounding that is puzzle/paradox free is better than one that isn't. Second, following Wilsch (2016), you might think that, while grounding is worldly in nature, what we're calling conceptual grounding is purely representational in nature. The idea is that the conjunction case targets logical/conceptual priority instead of grounding. ${ }^{21}$

sense, however, is that ontic covering law views of metaphysical explanation are no more plausible than their hybrid counterparts.

${ }^{21}$ Note that to establish that such claims involve logical/conceptual priority, however, isn't on its own to show that they don't involve ontological priority (grounding), as these claims may involve multiple senses of 'priority'. 
While I'm actually sympathetic to the no-conceptual-grounding proposal, for the purposes of this article I'll go with a more conservative response to the problem. The idea involves setting out further conditions on what it is to be a mechanistic model of a grounding relation, conditions that ensure that such representations are explanatorily apt. I propose to add a constraint concerning the form that grounding relations are represented as having. Recall the essential truths we discussed earlier that unify the metaphysical determination relations — call them the essential grounding truths. These truths connect metaphysical determination relations with grounding claims of a particular form. For example, the essential grounding truth characteristic of the determinate-determinable relation connects this relation to grounding claims that look like this: the fact that $\mathrm{x}$ has $\mathrm{P}$ grounds the fact that $\mathrm{x}$ has $\mathrm{Q}$. Now, suppose that $\Delta$ grounds $\mathrm{A}$ and that this grounding relation is an instance of a grounding mechanism. And suppose that there is a single metaphysical determination relation, $\mathrm{R}$, operative in this case rather than a chain of such relations. So $\mathrm{R}$ connects a constituent(s) of some fact among $\Delta$ with a constituent of $A$. The idea is that a representation of this grounding relation is a mechanistic model only if it does two things. First, it veridically represents $\mathrm{R}$ as being essentially connected to grounding claims of thus-and-so form. Second, it veridically represents the claim that $\Delta$ grounds A as being a grounding claim with that very form.

Let's return to the diamond case. Recall that I outlined three things that a mechanistic model of the grounding relation in this case will say. Given the proposal above, we need to add more content to the model. The model should also say that the grounding claim in question - the fact that the carbon atoms compose the diamond and are bonded and spatially arranged in thus-and-so way partially grounds the fact that the diamond is hard-has the same form as the grounding claims that mereological realization is essentially connected with. As mereological realization is essentially connected with grounding claims of the form "the fact that xs compose $y$ and have the Ps partially grounds the fact that y has Q", the model is veridical in so far as it contains this additional element. Hence, our model of the grounding relation in this case satisfies our additional constraint.

Now we can return to the Socrates case. Suppose that a model represents the grounding relation in this case, but it doesn't include information about the form of the grounding claims that set formation is essentially connected with. Given our additional constraint, this representation isn't a mechanistic model. And consider another model that represents the grounding relation in this case, one that says that the grounding claim in question - the fact that Socrates exists grounds the fact that Socrates exists or $\{$ Socrates $\}$ has $\mathrm{P}$ - has the same form as the grounding claims that set formation is essentially connected with. Given our additional constraint, this isn't 
a mechanistic model either, as set formation isn't essentially connected with grounding claims of the form "The fact that x exists grounds the fact that x exists or y has thus-and-so property". The same reasoning applies to other problem cases involving single metaphysical determination relations. And problem cases involving the chaining of metaphysical determination relations are subject to a similar treatment, but I won't go into the details here.

The moral of our discussion is this: we can resolve the problem I set out at the beginning of this section by adding a further condition on what it takes to be a mechanistic model of a grounding relation. Once this condition is in place, it's clear that cases like the Socrates one don't undermine the connection between mechanistic models of grounding relations and metaphysical explanation.

\subsection{Theoretical work for grounding-mechanical explanation}

A consideration that supports the grounding mechanism view is that the notion of grounding-mechanical explanation as I've characterized it seems to have a substantive theoretical role to play. Specifically, the grounding mechanism view gives us a straightforward way of evaluating the plausibility of grounding-theoretic theses. As such, grounding-mechanical explanation can serve as a useful diagnostic tool. Consider, for example, priority monism (about the concrete). ${ }^{22}$ Where the cosmos is the fusion of all concrete objects, cosmos facts are facts concerning the cosmos as a whole, and sub-cosmos facts are facts concerning the proper parts of the cosmos, priority monism as I understand it is the view that, for each sub-cosmos fact, there is a metaphysical explanation of why that fact obtains appealing to cosmos facts that ground it.

I don't wish to claim that all metaphysical explanations are grounding-mechanical explanations - it may be that some metaphysical explanations just don't involve grounding. ${ }^{23}$ Given what I've said so far, however, the following weaker claim seems fairly plausible: any metaphysical explanation that is backed by grounding — any

22 See Schaffer (2010) for the first in a series of papers defending priority monism.

23 Another conception of scientific explanation is the unificationist view according to which scientific explanation is a matter of deploying arguments that fit into a systemization that generates the largest possible number of conclusions using the smallest possible number of argument patterns - see Kitcher (1989) for further discussion. The correlate of this view concerning metaphysical explanation is worth exploring and may have nothing to do with grounding - see Kovacs forthcoming for discussion. In addition to the metaphysical correlate of the unificationist view of scientific explanation, it may be that there is a distinctive form of metaphysical explanation appealing to essences rather than grounding as well-see Glazier (forthcoming) for discussion. And there may be covering law metaphysical explanations that don't appeal to grounding. 
metaphysical explanation that proceeds by way of linking grounds to what they ground-is a grounding-mechanical explanation. ${ }^{24}$ Call this the mechanical backing claim. Provided that the mechanical backing claim is true, the priority monist discharges the explanatory burden of her thesis only if she can show "how the connection runs" between the cosmos facts and the sub-cosmos facts by identifying grounding mechanisms operative in these cases. So in this case one way to object to priority monism is to argue that there is no plausible story to tell about which metaphysical determination relations might be in play here.

The same considerations apply to other grounding-theoretic theses. Consider, for example, structuralism (about numbers) understood as the thesis that, for each fact concerning numbers, there is a metaphysical explanation of why that fact obtains appealing to facts about certain structures that (together with other non-arithmetic facts) ground it. Given the mechanical backing claim, the structuralist discharges the explanatory burden of her thesis only if she can identify grounding mechanisms operative in these cases. And one way to object to this view is to argue that there is no plausible story to tell about which metaphysical determination relations are operative here. ${ }^{25}$

To take another case, consider physicalism (about the mental) understood as the thesis that, for each mental fact, there is a metaphysical explanation of why that fact obtains appealing to physical facts that (together with other non-mental facts) ground it. Given the mechanical backing claim we can understand the so-called explanatory gap challenge to physicalism as follows: it strikes us that the connection between the mental and physical isn't mediated by grounding mechanisms. ${ }^{26}$

You might object to the mechanical backing claim - the claim that any groundingbacked metaphysical explanation is a grounding-mechanical explanation-as follows. Recall the notion of bare grounding, grounding relations that aren't instances of grounding mechanisms. Let's return to the putative case of bare grounding discussed earlier - the fact that $\mathrm{x}$ is red and the fact that $\mathrm{y}$ is round ground the conjunctive fact that $x$ is red and $y$ is round. Suppose that this is in fact a case of bare grounding. And suppose that the mechanical backing claim is true. Given these assumptions, it follows that, while the conjunctive fact is grounded by the conjunct facts, there is no grounding-backed metaphysical explanation of the former in terms of the latter. You

\footnotetext{
${ }^{24}$ For more on the notion of backing, see Kim (1994) and Ruben (2012, Ch. 6 \& 7).

25 See Shapiro (1997, Ch. 3) for more on structuralism.

26 See Levine (2001, Ch. 3) for a general discussion of the explanatory gap, and Schaffer (forthcoming) and Trogdon (2013b) for different takes on the relationship between grounding and explanatory gaps.
} 
might claim that there is such an explanation in this case. The culprit here, so the idea goes, is the mechanical backing claim.

We've already considered the idea that there is no conceptual grounding, so if any putative case of bare grounding is a case of conceptual grounding, perhaps it's not unreasonable to think that there just is no bare grounding. And if there is no bare grounding then there is no problem here. There are, however, putative cases of bare grounding that don't involve conceptual grounding. While my sense is that it's commonly assumed that grounding always takes place non-diachronically, there are reasons to reject this temporal condition-as A. Wilson (forthcoming) points out, the fact that you're a human as opposed to being Swampman seems to be grounded in your past causal history, as is the fact that you can refer to, say, Montana.

Supposing that these are genuine cases of grounding, the grounding relations in these cases aren't instances of grounding mechanisms, as metaphysical determination relations are non-diachronic in nature. ${ }^{27}$

One option, of course, is to deny that these putative cases of diachronic grounding really are cases of grounding. But, as with our discussion of the problem concerning cases in which pointing to grounding mechanisms doesn't illuminate how the grounding connection runs, there is a more conservative response available. The idea is just to say that, while cases of bare grounding don't involve grounding-backed metaphysical explanation, they potentially involve other sorts of explanation. A case can be made, for example, that the diachronic cases involve a different sort of metaphysical explanation (perhaps explanation by metaphysical subsumption), while the conceptual cases involve some form of non-metaphysical explanation (roughly, conceptual/logical explanation).

So I think that the mechanical backing claim is worth taking seriously. But even if you reject this claim, the considerations set out above show that groundingmechanical explanation is potentially important for evaluating grounding-theoretic theses in any case. If the mechanical backing claim is false then perhaps identifying metaphysical determination relations that connect constituents of cosmos facts with constituents of sub-cosmos facts isn't the only way for the priority monist to discharge the explanatory burden of her thesis. Nevertheless, doing so would be sufficient to discharge this burden. The same considerations apply to groundingtheoretic takes on structuralism, physicalism, and other theses. This suggests that the

27 One option is to drop the idea that all metaphysical determination relations are nondiachronic and argue that there are diachronic metaphysical determination relations operative in such cases. Just what these relations might be, however, isn't clear. It seems that it doesn't lie in the nature of causation, for example, to satisfy the grounding condition discussed earlier. 
advocates of grounding-theoretic theses would do well to consider the notion of grounding-mechanical explanation.

It's worth noting that there is a slightly different way to approach the explanatory burden of grounding-theoretic theses like priority monism. But on his approach we get the conclusion that grounding-mechanical explanation is potentially useful as well. Following Sider (manuscript, Ch. 4), you might think that a condition of adequacy for priority monism (as a grounding-theoretic thesis) is that the priority monist accounts for what enables the cosmos facts to ground the sub-cosmos facts. Sider suggests that if there is no such account to be had then priority monism is magical in the sense that nothing about the cosmos facts or sub-cosmos facts explains why the former are able to ground the latter. Another possibility is that the thesis in this case is occult - while there are considerations about these facts that explain why the former are able to ground the later, these considerations are hidden in that they're beyond our ken. Neither option is attractive. Call this the grounding aptness constraint on priority monism. ${ }^{28}$

How might the priority monist meet this constraint? Establishing what grounding mechanisms are operative in a case of grounding gives us purchase not only on "how the connection runs" between the grounding and the grounded but also why the grounding facts are up to the job of grounding what they ground. To return to a case that we've already discussed, by peeking inside the fact that thus-and-so nucleotides compose $\mathrm{x}$ and have thus-and-so properties and the fact that $\mathrm{x}$ is a gene we can see why the first fact is able to partially ground the second, as we find that constituents of the first fact are connected via a chain of metaphysical determination relations to a constituent of the second. Similar considerations apply in the case of causal mechanisms. Given the system view of causal mechanisms, a causal-mechanical explanation accounts for a system's capacity to produce certain outputs given certain inputs.

Given the discussion above, one way for the priority monist to satisfy the grounding aptness constraint is to provide certain grounding-mechanical explanations, specifically models of cosmos-to-sub-cosmos grounding relations according to which they're instances of particular grounding mechanisms. The same applies to grounding-theoretic versions of other theses like structuralism and physicalism as well. $^{29}$

\footnotetext{
28 See Sider (2008) and (2011, Ch. 8) for related discussion.

${ }^{29} \mathrm{I}$ take it that questions about how the connection runs in a case of grounding and questions about why grounding facts are able to ground what they ground can potentially come apart. It seems, for example, that the interventionist claims explored by Schaffer
} 


\section{Conclusion}

In this paper I argued that there is an important similarity between causation and grounding. I argued in particular that there are grounding mechanisms in addition to causal mechanisms, and that the former contribute to metaphysical explanations in a way that corresponds to how the latter contribute to scientific explanations. I also argued that grounding-mechanical explanation has an important role to play in evaluating philosophical theses. Returning to the idea that I opened the paper with, I hope to have shed further light on the nature of grounding by showing how it resembles causation.

For a final thought I turn to skepticism about grounding. My primary target in this paper isn't the grounding skeptic_-it's incumbent upon the proponent of grounding to provide comprehensive responses to skeptical challenges to grounding, but I leave this task for another day. Nevertheless, it's worth noting that what I've said has consequences for J. Wilson's (2014) skepticism about grounding in particular. ${ }^{30}$ Wilson's skeptical case is multifaceted, but one strand of her argument concerns the connection between grounding and explanation. Some proponents of grounding claim that it plays an indispensible role in certain explanations. ${ }^{31}$ Wilson objects, arguing that claims to the effect that this grounds that are explanatory only when supplemented with claims about the instantiation of more fine-grained determination relations. I have in effect argued that even if her claim about the supplementation of grounding claims is correct we have reason to think that grounding plays an indispensable role in certain metaphysical explanations. Indeed, I've argued that the

(2016a) and A. Wilson (forthcoming) speak to the first question but not necessarily the second, and the essentialist claims explored by Dasgupta (2015), Correia (2013), and Trogdon (2013b) speak to the second question but not necessarily the first. And I take it that both of these questions can come apart from the question about what grounds the facts about what grounds what. It seems, for example, that what I've said about grounding mechanisms speaks to the how the connection runs question but not necessarily the what grounds grounding question, and the "super-internalist" proposals offered by Bennett (2011) and deRosset (2013) speak to the what grounds grounding question but not necessarily the grounding ability question.

${ }^{30}$ For further skeptical challenges to grounding, see Daly (2012), Hofweber (2009), Koslicki (2015), Kovacs (forthcoming), Miller and Norton (forthcoming), and J. Wilson (2016).

31 See, e.g., Audi (2012), Fine (2012), and Schaffer (2009). 
close relationship between grounding and certain fine-grained determination relations actually counts in favor of the idea that grounding plays such a role. ${ }^{32}$

\section{References}

Aizawa, K. and C. Gillett (eds.) 2016a. Scientific Composition and Metaphysical Ground. Palgrave.

_ 2016b. "Vertical Relations in Science, Philosophy, and the World: Understanding the New Debates of Verticality." In Aizawa and Gillett.

Andersen, H. 2011. "Mechanisms, Laws, and Regularities," Philosophy of Science 78: $325-331$.

Armstrong, D. M. 1997. A World of States of Affairs. Cambridge UP.

Audi, P. 2012. "Grounding: Toward a Theory of the In-Virtue-Of Relation," Journal of Philosophy 109: 685-711.

Baysan, U. 2015. "Realization Relations in Metaphysics," Minds and Machines 25: 247260.

Beatty, J. 1995. “The Evolutionary Contingency Thesis.” In (eds.) G. Wolters and J. G. Lennox, Concepts, Theories, and Rationality in the Biological Sciences. University of Pittsburgh Press.

Bechtel, W. and A. Abrahamsen. 2005. "Explanation: A Mechanist Alternative," Studies in History and Philosophy of Science Part C: Studies in History and Philosophy of Biological and Biomedical Sciences 36: 421-441.

Bennett, K. 2011. "By our Bootstraps,” Philosophical Perspectives 25: 27-41.

Bernstein, S. Forthcoming. "Grounding is not Causation," Philosophical Perspectives.

Chalmers, D., D. Manley, and R. Wasserman (eds.) 2009. Metametaphysics, Oxford UP.

32 I presented versions of this paper at the Metaphysical Explanation Workshop at the University of Gothenburg (September 2016) and the Grounding and Explanation Workshop at the University of Agder (March 2016). I also presented related material at the Truth and Grounds Conference in Ascona Switzerland (May 2015), the First Veritas Philosophy Conference at Yonsei University (June 2015), the University of Florida (April 2014), the American Philosophical Association Central Division (February 2014), and the 1st Annual Junior Metaphysics Workshop at Western Michigan University (November 2013). For helpful feedback I wish to thank my colleagues at Virginia Tech, as well as Ken Aizawa, Ricki Bliss, Einar Bohn, Ben Caplan, Sam Cowling, Shamik Dasgupta, Louis deRosset, Tim Fuller, Kathrin Koslicki, David Kovacs, Jon Litland, Anna-Sofia Maurin, Tristram McPerherson, Michael Raven, Gonzalo Rodriguez-Pereyra, Jonathan Schaffer, Alexander Skiles, Giuliano Torrengo, Tobias Wilsch, Alastair Wilson, Gene Witmer, and anonymous referees. 
Clark, M. 2015. “A Puzzle about Partial Grounding,” Thought 4: 189-197.

Correia, F. 2013. “Metaphysical Grounds and Essence.” In Hoeltje et al.

Correia, F. and B. Schnieder (eds.) 2012. Metaphysical Grounding: Understanding the Structure of Reality. Cambridge UP.

Craver, C. 2006. “When Mechanistic Models Explain,” Synthese 153: 355-376.

— 2007. Explaining the Brain: Mechanisms and the Mosaic Unity of Neuroscience. Oxford UP.

Craver, C. \& M. Kaiser. 2013. "Mechanisms and Laws: Clarifying the Debate.” In (eds.) Hsiang-Ke Chao et al, Mechanism and Causality in Biology and Economics. Springer.

Cummins, R. 2000. “'How Does it Work?’ Versus 'What are the Laws?': Two Conceptions of Psychological Explanation.” In (eds.) F. Keil and R. Wilson, Explanation and Cognition. MIT Press.

Daly, C. 2012. "Skepticism about Grounding." In Correia and Schnieder.

Dasgupta, S. 2015. “The Possibility of Physicalism," Journal of Philosophy 111: 557592.

deRosset, L. 2013. “Grounding Explanations,” Philosophers' Imprint 13: 1-26.

Doepke, F. 1999. The Kinds of Things: A Theory of Personal Identity Based on Transcendental Argument. Open Court.

Dowe, P. 2000. Physical Causation. Cambridge UP.

Fine, K. 1982. "First-Order Modal Theories III-Facts," Synthese 53: 43-122.

— 1994. "Essence and Modality," Philosophical Perspectives 8: 1-16.

— 2010a. “Towards a Theory of Part," Journal of Philosophy 107: 559-589.

_ 2010b. "Some Puzzles of Ground," Notre Dame Journal of Formal Logic 51: 97118.

— 2012. "A Guide to Ground.” In Correia and Schnieder.

Franklin-Hall, L. R. 2016. "New Mechanistic Explanation and the Need for Explanatory Constraints.” In Aizawa and Gillett.

Gillett, C. 2007. "Understanding the New Reductionism: the Metaphysics of Science and Compositional Reduction," Journal of Philosophy 104: 193-216.

Glazier, M. Forthcoming. “Essentialist Explanation,” Philosophical Studies.

Glennan, S. 2002. "Rethinking Mechanistic Explanation,” Philosophy of Science 69: S342-S353. 
- 2005. "Modeling Mechanisms," Studies in History and Philosophy of Biological and Biomedical Sciences 36: 443-464.

Hoeltje, M., B. Schnieder, and A. Steinberg (eds.) 2013. Varieties of Dependence. Philosophia Verlag.

Hofweber, T. “Ambitious, Yet Modest, Metaphysics.” In Chalmers et al.

Kaplan, D. M. and Bechtel, W. 2011. "Dynamical Models: An Alternative or Complement to Mechanistic Explanations?” Topics in Cognitive Science 3: 438-44.

Kim, J. 1994. "Explanatory Realism, Causal Realism, and Explanatory Exclusion.” In his Essays in the Metaphysics of Mind. Oxford UP.

Kitcher, P. 1989. "Explanatory Unification and the Causal Structure of the World." In (eds.) P. Kitcher and W. Salmon, Scientific Explanation. Minnesota UP.

Kment, B. 2014. Modality and Explanatory Reasoning. Oxford UP.

Koslicki, K. 2015. "The Coarse-Grainedness of Grounding.” In (eds.) K. Bennett and D. Zimmerman, Oxford Studies in Metaphysics, Vol. 9. Oxford UP.

— 2016. "Where Grounding and Causation Part Ways: Comments on Jonathan Schaffer," Philosophical Studies 173: 101-112.

Kovacs, D. Forthcoming. "Grounding and the Argument from Explanatoriness," Philosophical Studies.

Levine, J. 2001. Purple Haze. Oxford UP.

Lewis, D. 1991. Parts of Classes. Blackwell.

Litland, J. 2015. "Grounding, Explanation, and the Limit of Internality," Philosophical Review 124: 481-532.

Machamer, P. K., Darden, L., \& Craver, C. F. 2000. “Thinking about Mechanisms," Philosophy of Science 57: 1-25.

Miller, K. \& J. Norton. Forthcoming. "Grounding: it's (probably) all in the head," Philosophical Studies.

Mitchell, S. 2000. "Dimensions of Scientific Law," Philosophy of Science 67: 242-265.

Polger, T. 2010. "Mechanisms and Explanatory Realization Relations," Synthese 177: 193-212.

Railton, P. 1978. "A Deductive-Nomological Model of Probabilistic Explanation," Philosophy of Science 45: 206-226.

Ruben, D. 2012. Explaining Explanation, $2^{\text {nd }}$ edition. Paradigm. 
Salmon, W. 1984. Scientific Explanation and the Causal Structure of the World. Princeton UP.

— 1989. Four Decades of Scientific Explanation. Pittsburgh UP.

Schaffer, J. 2009. “On What Grounds What.” In Chalmers et al.

— 2010. "Monism: the Priority of the Whole," Philosophical Review 119: 31-76.

— 2012. "Grounding, Transitivity, and Contrastivity." In Correia and Schnieder.

_ 2016a. "Grounding in the Image of Causation," Philosopbical Studies 173: 49-100.

— 2016b. “Ground Rules: Lessons from Wilson.” In Aizawa and Gillett.

— Forthcoming. "The Ground Between the Gaps," Philosophers' Imprint.

Shapiro, S. 1997. Philosophy of Mathematics: Structure and Ontology. Oxford UP.

Sider, T. 2008. "Monism and Statespace Structure." In (ed.) Robin Le Poidevin, Being: Developments in Contemporary Metaphysics. Cambridge UP.

— 2011. Writing the Book of the World. Oxford UP.

— Manuscript. The Tools of Metaphysics and the Metaphysics of Science.

Trogdon, K. 2013a. “An Introduction to Grounding.” In Hoeltje et al.

_ 2013b. “Grounding: Necessary or Contingent?” Pacific Philosophical Quarterly 94: 465-485.

Wasserman, R. 2015. "Material Constitution.” In (eds.) N. Zalta, The Stanford Encyclopedia of Philosophy.

Wilsch, T. 2015. "The Nomological Account of Ground," Philosophical Studies 172: 3293-3312.

— 2016. "The Deductive-Nomological Account of Metaphysical Explanation," Australasian Journal of Philosopby 94: 1-23.

Wilson, A. Forthcoming. "Metaphysical Causation," Nous.

Wilson, J. 1999. "How Superduper Does a Physicalist Supervenience Need to Be?" The Philosophical Quarterly 49: 33-52.

— 2014. "No Work for a Theory of Grounding," Inquiry 57: 535-579.

— 2016. "The Unity and Priority Arguments for Grounding." In Aizawa and Gillett.

— 2017. "Determinables and Determinates." In (eds.) N. Zalta, The Stanford Encyclopedia of Philosophy. 
